\title{
*INTERNATIONAL FEDERATION OF CATHOLIC UNIVERSITIES (IFCU) FEDERATION INTERNATIONALE DES UNIVERSITES CATHOLIQUES (FIUC)
}

51 , rue Orfila

75020 Paris (France)

Tel: (33-1) 4797-2660

Fax: (33-1) 4797-2942

President: Michel Falise, Rector, Fédération

universitaire et polytechnic de Lille

Secretary-General: Marc Caudron

An international association by Vatican decree and Apostolic letters. Full membership comprises universities with three or more faculties; Associate membership includes universities, colleges or institutes with two or less faculties. The purpose of the Federation is "to work for the constant progress of knowledge and for the development of a more just and human society in the light of the Christian faith and the Gospel". Its functions are: to promote collective reflection by providing a world forum in a variety of meetings; to maintain representation on behalf of Catholic universities through liaison with international bodies; to contribute to an effective collaboration among institutions by various exchanges; and to provide information to members by selected means of communications. IFCU activities take place at four levels: international or world wide; regional or continental; sectoral or by faculties/schools; and interdisciplinary through research projects organized by the Centre for Coordination of Research (Palazzo Frascara, Università Gregoriana, Piazza della Pilotta 4, 00187 Roma). Topics of study include: university identity and government; cultural issues; economic questions; social matters; and ethical problems.

Principal publications in English include: Image of Main in Human Rights Legislation; The Expectations of Youth and the Catholic University; Human Life: Its Beginning and Development; Faith and Culture: The Role of the Catholic University; Multinational Enterprises and National Policies. In Italian: Una pratica cristiana dell' Economia; La Pace: Sfida all'Università Cattolica.
Une Association internationale érigée par décret du Vatican et lettre apostolique. Les membres de plein droit sont les universités ayant trois facultés ou équivalent; les mem-bres associés sont les universités, collèges ou institutions ayant deux facultés ou moins. La finalité de la Fédération est "de contribuer, à la lumière de la foi chrétienne et grâce au ferment de l'Evangile, au progrès du Savoir et à l'élaboration d'un monde plus juste et plus humain". Elle a ainsi une triple mission, intellectuelle, sociale et spirituelle. Les fonctions de la FIUC sont: la promotion de la réflexion collective dans divers forums et assemblées; la représentation au nom des universités catholiques par des liaisons auprès des organismes internationaux; la collaboration efficace par un ensemble d'échanges entre les institutions; la diffusion d'informations aux membres par divers moyens de communication. Les activités de la Fédération se situent à quatre niveaux: international ou mondial; régional ou national; sectoriel ou par disciplines; et interdisciplinaire à travers des projets de recherche organisés par le Centre de Coordination de la Recherche (Palazzo Frascara,Université Grégorienne, Piazzadella Pilotta 4, 00187 Roma). Les sujets d'étude se regroupent principalement autour des thèmes suivants: identité et gouvernement de l'université; enjeux culturels; questions économiques; affaires sociales; problèmes éthiques.

Parmi les publications principales en français: "Les Attentes de la Jeunesse et l'Université catholique"; "Le Respect et la Liberté, Droits de l'Homme, Raison et Foi"; "Débuts biologiques de la Vie humaine"; "Foi et Culture, le Rôle de l' Université catholique"; " "Crise économique et Mutation des Valeurs". En italien: "La Pace: Sfida all'Università Cattolica”. 